\title{
The Moment Properties of Order, Reversed Order and Upper Record Statistics for the Power Ailamujia Distribution
}

\author{
FARRUKH JAMAL \\ Department of Statistics, The Islamia University of Bahawalpur, Punjab 63100, \\ PAKISTAN \\ CHRISTOPHE CHESNEAU \\ Department of Mathematics, Université de Caen, LMNO, Campus II, Science 3, Caen 14032, \\ FRANCE
}

\begin{abstract}
The power Ailamujia distribution has been successfully developed in statistics, both theoretically and practically, performing well in the fitting of various types of data. This paper investigates the moment properties of the associated order, reversed order and upper record statistics, which are indeed unexplored aspects of this distribution. In particular, the exact expressions for the single moments of the order and reversed order statistics are provided. Some recurrence relationships for both single and product moments for the order and upper record statistics are proved. For additional goals, certain joint distributions are also given.
\end{abstract}

Key-Words: Power Ailmujia distribution; order statistics; reversed order statistics; upper record statistics; recurrence relationships.

Received: May 13, 2021. Revised: October 18, 2021. Accepted: October 30, 2021. Published: November 18, 2021.

\section{Introduction}

The ordering of observed values is a common operation in many aspects of our lives. In general, we might be interested in organizing various things: an instructor might be interested in classifying students based on their test grades, a sports coach might be interested in assembling a sports team based on the best performance of the candidate players, an item can be assigned to the customer with the highest bid, etc. For an understanding of the distributions and the characteristics linked to these order values, modeling through random variables is necessary. The concept of ordered random variables is vast and plays a major role in simple statistical measures like the range, the median and the percentiles etc. The ordered random variables are based upon different models depending upon how the ordering is being done. For instance, when observations in a sample are arranged in increasing order, then we use order statistics, which is the oldest model of ordered random variables.

The distributional and moment properties of ordered random variables from a particular survival distribution, the power Ailamujia (PA) distribution, are discussed in this paper. In order to inspire this goal, a look back at the PA distribution is needed. The exponential, Lomax, and gamma distributions are the most widely used in reliability data processing, but they are not always the best in all cases. Therefore, many modern distributions for different types of engineering applications have been introduced in recent years. The
Ailamujia distribution, introduced by [1], is one of the simplest and most promising distributions in this regard, constituting a flexible right-skewed alternative. The power transformation process is used to create the PA distribution, which is an expansion of the Ailamujia distribution. It was conjointly developed in the works of [2] and [3]. The characteristics of these works are described below. To begin, a random variable $X$ has the PA distribution if its probability density function (pdf) has the following form:

$$
f(x)=\theta^{2} \beta x^{2 \beta-1} e^{-\theta x^{\beta}}, \quad x>0,
$$

and $f(x)=0$ for $x \leq 0$, where $\theta$ and $\beta$ are two strictly positive tuning parameters, and the related cumulative distribution function (cdf) can be expressed as

$$
F(x)=1-\left(1+\theta x^{\beta}\right) e^{-\theta x^{\beta}}, \quad x>0,
$$

where $F(x)=0$ for $x \leq 0$. The standard properties of the PA distribution, such as moments (ordinary and incomplete), mode, median, Shanon's entropy and the distributional based on order statistics, are studied in [2]. For the development of the PA model, the maximum likelihood approach is used. It is shown that the PA model accommodates two distinct types of medical data. In [3], complementary properties such as stochastic orders, probability weighted moments, stress-strength reliability coefficient, and Tsallis' entropy are demonstrated. In addition, some general PA classes of distributions are created. Then, the 
PA model was developed to consider two different types of data: full data and data with censorship. A goodness-of-fit statistical test that allows the processing of right-censored data is built, in particular. The researchers then looked at four separate data sets, two of which were related to the Covid-19 outbreak.

Despite these in-depth investigations, certain distributional and moment properties of order, reversed order and upper record statistics based on the PA distribution remain unknown, which is the subject of this article. The use of moments of order statistics is well documented in mathematical literature. They can be used in mathematical modeling, statistical inferences, decision methods, and nonparametric statistics, to name a few. For certain types of distributions in general, or for some particular distributions, several authors have developed many recurrence formulas for diverse moments of order statistics. See [4], [5], [6] and [7]. Moments of order statistics are often used to obtain the best linear unbiased estimates for some parameters of distributions based on complete and 'type-II censored' samples. In this regard, we refer the reader to [8] and [9]. Similar observations can be made with the upper record statistics introduced by [10]; their moments can be useful in a variety of statistical applications, such as sporting and sporting activities, oil and gas studies, and bioscience. See [11], [12] and [13], and the references therein.

The following portions make up the article. Section 2 is devoted to diverse moments of the order and reversed order statistics for the PA distribution, discussing expressions and recurrence relationships. The moments of the upper record statistics for the PA distribution are established in Section 3 .

\section{Order statistics}

[14. Chapter 2] contains all of the general formulas included in this portion, which remains quite classical. The contributions consist of applying them to the PA distribution via various approaches.

\subsection{Univariate case}

Some general properties of order statistics are developed below. Let $X_{1}, X_{2}, \ldots, X_{n}$ be $n$ independent random variables with a common distribution. The corresponding cdf and pdf of this common distribution are denoted by $F(x)$ and $f(x)$, respectively. Now, we arrange these random variables in increasing order of magnitude to obtain ordered random variables denoted by $X_{1: n}, X_{2: n}, \ldots, X_{n: n}$ such that $P\left(X_{1: n} \leq X_{2: n} \leq \ldots \leq X_{n: n}\right)=1$. These random variables are called order statistics. In full generality, for any $r=1,2, \ldots, n$, the pdf of $X_{r: n}$ is given as

$$
\begin{aligned}
& f_{r: n}(x)=C_{r: n} f(x)[F(x)]^{r-1}[1-F(x)]^{n-r}, \\
& x \in \mathbb{R},
\end{aligned}
$$

where $C_{r: n}=n ! /[(n-r) !(r-1) !]$. In particular, if $r=1$, this pdf reduces to $f_{1: n}(x)=n f(x)[1-$ $F(x)]^{n-1}$ and, if $r=n$, it reduces to $f_{n: n}(x)=$ $n f(x) F(x)^{n-1}$. In the context of the PA distribution, it comes.

$$
\begin{aligned}
& f_{r: n}(x)=C_{r: n} \theta^{2} \beta x^{2 \beta-1}\left[1-\left(1+\theta x^{\beta}\right) e^{-\theta x^{\beta}}\right]^{r-1} \times \\
& \left(1+\theta x^{\beta}\right)^{n-r} e^{-(n-r+1) \theta x^{\beta}}, \quad x>0,
\end{aligned}
$$

and $f_{r: n}(x)=0$ for $x \leq 0$.

Also, for the 'two extreme order statistics', we get

$f_{1: n}(x)=n \theta^{2} \beta x^{2 \beta-1}\left(1+\theta x^{\beta}\right)^{n-1} e^{-\theta x^{\beta} n}, \quad x>0$

and $f_{1: n}(x)=0$ for $x \leq 0$, and

$f_{n: n}(x)=n \theta^{2} \beta x^{2 \beta-1}\left[1-\left(1+\theta x^{\beta}\right) e^{-\theta x^{\beta}}\right]^{n-1} e^{-\theta x^{\beta}}$, $x>0$,

and $f_{n: n}(x)=0$ for $x \leq 0$.

The exact expression of the $p$ th moment of $X_{r: n}$ is investigated in the following result.

Proposition 2.1 Let $p$ be a positive integer and $\mu_{r: n}^{p}=E\left(X_{r: n}^{p}\right)$ be the pth moment of $X_{r: n}$. Then, $\mu_{r: n}^{p}$ can be expressed as

$$
\begin{aligned}
& \mu_{r: n}^{p}=C_{r: n} \theta^{-p / \beta} \sum_{k=0}^{r-1} \sum_{v=0}^{k+n-r}\left(\begin{array}{c}
r-1 \\
k
\end{array}\right)\left(\begin{array}{c}
k+n-r \\
v
\end{array}\right) \times \\
& (-1)^{k} \frac{1}{(k+n-r+1)^{p / \beta+2+v}} \Gamma\left(\frac{p}{\beta}+2+v\right),
\end{aligned}
$$

where $\left(\begin{array}{l}b \\ a\end{array}\right)$ denotes the usual binomial coefficient and $\Gamma(x)$ denotes the usual gamma function.

Proof: First, by Equation (1), we have

$$
\begin{aligned}
& \mu_{r: n}^{p}=\int_{-\infty}^{+\infty} x^{p} f_{r: n}(x) d x \\
& =C_{r: n} \theta^{2} \beta \int_{0}^{+\infty} x^{p+2 \beta-1}\left[1-\left(1+\theta x^{\beta}\right) e^{-\theta x^{\beta}}\right]^{r-1} \times \\
& \left(1+\theta x^{\beta}\right)^{n-r} e^{-(n-r+1) \theta x^{\beta}} d x .
\end{aligned}
$$

Now, we need to develop at the best the power function of the form $(1+a)^{b}$. By applying the binomial formula twice in a row, we get

$$
\begin{aligned}
& {\left[1-\left(1+\theta x^{\beta}\right) e^{-\theta x^{\beta}}\right]^{r-1}\left(1+\theta x^{\beta}\right)^{n-r} e^{-(n-r+1) \theta x^{\beta}}} \\
& =\sum_{k=0}^{r-1}\left(\begin{array}{c}
r-1 \\
k
\end{array}\right)(-1)^{k}\left(1+\theta x^{\beta}\right)^{k+n-r} e^{-(k+n-r+1) \theta x^{\beta}} \\
& =\sum_{k=0}^{r-1} \sum_{v=0}^{k+n-r}\left(\begin{array}{c}
r-1 \\
k
\end{array}\right)\left(\begin{array}{c}
k+n-r \\
v
\end{array}\right)(-1)^{k} \theta^{v} \times \\
& x^{\beta v} e^{-(k+n-r+1) \theta x^{\beta}} .
\end{aligned}
$$


Therefore, upon integration of the suitable function, it comes

$$
\begin{aligned}
& \mu_{r: n}^{p}=C_{r: n} \theta^{2} \beta \sum_{k=0}^{r-1} \sum_{v=0}^{k+n-r}\left(\begin{array}{c}
r-1 \\
k
\end{array}\right)\left(\begin{array}{c}
k+n-r \\
v
\end{array}\right) \times \\
& (-1)^{k} \theta^{v} \int_{0}^{+\infty} x^{p+(2+v) \beta-1} e^{-(k+n-r+1) \theta x^{\beta}} d x .
\end{aligned}
$$

Making the transformations $y=\theta x^{\beta}$ and $z=(k+$ $n-r+1) y$ in a row, we obtain

$$
\begin{aligned}
& \mu_{r: n}^{p}=C_{r: n} \theta^{-p / \beta} \sum_{k=0}^{r-1} \sum_{v=0}^{k+n-r}\left(\begin{array}{c}
r-1 \\
k
\end{array}\right)\left(\begin{array}{c}
k+n-r \\
v
\end{array}\right) \times \\
& (-1)^{k} \int_{0}^{+\infty} y^{p / \beta+1+v} e^{-(k+n-r+1) y} d y \\
& =C_{r: n} \theta^{-p / \beta} \sum_{k=0}^{r-1} \sum_{v=0}^{k+n-r}\left(\begin{array}{c}
r-1 \\
k
\end{array}\right)\left(\begin{array}{c}
k+n-r \\
v
\end{array}\right) \times \\
& (-1)^{k} \frac{1}{(k+n-r+1)^{p / \beta+2+v}} \Gamma\left(\frac{p}{\beta}+2+v\right) .
\end{aligned}
$$

The desired result is established.

Eventually, we can extract the gamma term of the sum in the expression of $\mu_{r: n}^{p}$ by using the formula $\Gamma(p / \beta+2+v)=\Gamma(p / \beta) \prod_{j=1}^{v+2}(p / \beta+2+v-j)$.

Also, from Proposition 2.1, we derive the mean of $X_{r: n}$ as

$$
\begin{aligned}
& \mu_{r: n}^{1}=C_{r: n} \theta^{-1 / \beta} \sum_{k=0}^{r-1} \sum_{v=0}^{k+n-r}\left(\begin{array}{c}
r-1 \\
k
\end{array}\right)\left(\begin{array}{c}
k+n-r \\
v
\end{array}\right) \times \\
& (-1)^{k} \frac{1}{(k+n-r+1)^{1 / \beta+2+v}} \Gamma\left(\frac{1}{\beta}+2+v\right)
\end{aligned}
$$

and the variance of $X_{r: n}$ as

$$
\begin{aligned}
& V=C_{r: n} \theta^{-2 / \beta} \sum_{k=0}^{r-1} \sum_{v=0}^{k+n-r}\left(\begin{array}{c}
r-1 \\
k
\end{array}\right)\left(\begin{array}{c}
k+n-r \\
v
\end{array}\right) \times \\
& (-1)^{k} \frac{1}{(k+n-r+1)^{2 / \beta+2+v}} \Gamma\left(\frac{2}{\beta}+2+v\right) \\
& -\left[\mu_{r: n}^{1}\right]^{2} .
\end{aligned}
$$

Other moments measures (moments skewness measures, moments kurtosis measures...) can be obtained in a similar fashion.

The following result shows that the moments are linked by a manageable recurrence relationship.

Proposition 2.2 The following recurrence relationship holds:

$$
\mu_{r: n}^{p}=\mu_{r-1: n}^{p}+\frac{p}{n-r+1} \frac{1}{\theta^{2} \beta}\left[\mu_{r: n}^{p-2 \beta}+\theta \mu_{r: n}^{p-\beta}\right] .
$$

Proof: In full generality in terms of notation, it follows from [14, Theorem 2.10, Equation (2.57)] that

$$
\begin{aligned}
& \mu_{r: n}^{p}-\mu_{r-1: n}^{p}=C_{r: n} \frac{p}{n-r+1} \int_{-\infty}^{+\infty} x^{p-1} \times \\
& {[F(x)]^{r-1}[1-F(x)]^{n-r+1} d x .}
\end{aligned}
$$

Now, let us notice that, in the context of the PA distribution, we have $\theta^{2} \beta x^{2 \beta-1}[1-F(x)]=(1+$ $\left.\theta x^{\beta}\right) f(x)$ for $x>0$, implying that

$$
\begin{aligned}
& x^{p-1}[F(x)]^{r-1}[1-F(x)]^{n-r+1}=\frac{1}{\theta^{2} \beta} x^{p-2 \beta} \times \\
& {[F(x)]^{r-1}[1-F(x)]^{n-r}\left\{\theta^{2} \beta x^{2 \beta-1}[1-F(x)]\right\}} \\
& =\frac{1}{\theta^{2} \beta} x^{p-2 \beta}[F(x)]^{r-1}[1-F(x)]^{n-r}\left(1+\theta x^{\beta}\right) f(x) \\
& =\frac{1}{\theta^{2} \beta}\left\{x^{p-2 \beta}[F(x)]^{r-1}[1-F(x)]^{n-r} f(x)\right. \\
& \left.+\theta x^{p-\beta}[F(x)]^{r-1}[1-F(x)]^{n-r} f(x)\right\} \\
& =\frac{1}{\theta^{2} \beta}\left\{x^{p-2 \beta} C_{r: n}^{-1} f_{r: n}(x) d x+\theta x^{p-\beta} C_{r: n}^{-1} f_{r: n}(x)\right\} .
\end{aligned}
$$

Hence, by combining Equations (2) and (3), we obtain

$$
\begin{aligned}
& \mu_{r: n}^{p}-\mu_{r-1: n}^{p}= \\
& \frac{p}{n-r+1} \frac{1}{\theta^{2} \beta}\left[\int_{0}^{+\infty} x^{p-2 \beta} f_{r: n}(x) d x\right. \\
& \left.+\theta \int_{0}^{+\infty} x^{p-\beta} f_{r: n}(x) d x\right] \\
& =\frac{p}{n-r+1} \frac{1}{\theta^{2} \beta}\left[\mu_{r: n}^{p-2 \beta}+\theta \mu_{r: n}^{p-\beta}\right] .
\end{aligned}
$$

The desired result is established.

The relationships among moments of order statistics proved in Proposition 2.2 enable us to compute certain moments of higher order statistics on the basis of lower order statistics and/or on the basis of lower order moments.

\subsection{Bivariate case}

We now focus on the bivariate case of the order statistics. First, the general expression of the joint pdf of $\left(X_{r: n}, X_{s: n}\right)$ for $r \leq s$ is given as

$$
\begin{aligned}
& f_{r, s: n}\left(x_{r}, x_{s}\right)=C_{r, s: n} f\left(x_{r}\right) f\left(x_{s}\right)\left[F\left(x_{r}\right)\right]^{r-1} \times \\
& {\left[F\left(x_{s}\right)-F\left(x_{r}\right)\right]^{s-r-1}\left[1-F\left(x_{s}\right)\right]^{n-s}, \quad x_{r}<x_{s},}
\end{aligned}
$$


with $\left(x_{r}, x_{s}\right) \in \mathbb{R}^{2}$, where $C_{r, s: n}=n ! /[(r-1) !(s-$ $r-1) !(n-s) !]$. In the context of the PA distribution, it comes

$$
\begin{aligned}
& f_{r, s: n}\left(x_{r}, x_{s}\right)=C_{r, s: n} \theta^{4} \beta^{2} x_{r}^{2 \beta-1} x_{s}^{2 \beta-1} e^{-\theta x_{r}^{\beta}} \times \\
& {\left[1-\left(1+\theta x_{r}^{\beta}\right) e^{-\theta x_{r}^{\beta}}\right]^{r-1}} \\
& \times\left[\left(1+\theta x_{r}^{\beta}\right) e^{-\theta x_{r}^{\beta}}-\left(1+\theta x_{s}^{\beta}\right) e^{-\theta x_{s}^{\beta}}\right]^{s-r-1} \times \\
& \left(1+\theta x_{s}^{\beta}\right)^{n-s} e^{-(n-s+1) \theta x_{s}^{\beta}}, \\
& x_{s}>x_{r}>0,
\end{aligned}
$$

and $f_{r, s: n}\left(x_{r}, x_{s}\right)=0$ when $x_{s}>x_{r}>0$ is not satisfied.

In particular, by taking $r=1$ and $s=n$ the joint $\operatorname{pdf}$ of $\left(X_{1: n}, X_{n: n}\right)$ is readily written as

$f_{1, n: n}\left(x_{1}, x_{n}\right)=n(n-1) \theta^{4} \beta^{2} x_{1}^{2 \beta-1} x_{n}^{2 \beta-1} \times$

$e^{-\theta x_{1}^{\beta}} e^{-\theta x_{n}^{\beta}}\left[\left(1+\theta x_{1}^{\beta}\right) e^{-\theta x_{1}^{\beta}}-\left(1+\theta x_{n}^{\beta}\right) e^{-\theta x_{n}^{\beta}}\right]^{n-2}$, $x_{n}>x_{1}>0$,

and $f_{1, n: n}\left(x_{1}, x_{n}\right)=0$ when $x_{n}>x_{1}>0$ is not satisfied.

These functions, which are novel in the literature, can be used to model a wide range of bidimensional phenomena, particularly those found in random systems.

The following result is about a recurrence relationship involving the product moments of order statistics for the PA distribution.

Proposition 2.3 Let $p$ and $q$ be positive integers and $\mu_{r, s: n}^{p, q}=E\left(X_{r: n}^{p} X_{s: n}^{q}\right)$ be the $(p, q)$ th product moment of $\left(X_{r: n}, X_{s: n}\right)$. Then, $\mu_{r, s: n}^{p, q}$ satisfies the following relationship:

$$
\begin{aligned}
& \mu_{r, s: n}^{p, q}= \\
& \mu_{r, s-1: n}^{p, q}+\frac{q}{n-s+1} \frac{1}{\theta^{2} \beta}\left[\mu_{r, s: n}^{p, q-2 \beta}+\theta \mu_{r, s: n}^{p, q-\beta}\right] .
\end{aligned}
$$

Proof: In full generality, it follows from [14, Theorem 2.10, Equation (2.58)] that

$$
\begin{aligned}
& \mu_{r, s: n}^{p, q}-\mu_{r, s-1: n}^{p, q}=C_{r, s: n} \frac{q}{n-s+1} \times \\
& \int_{-\infty}^{+\infty}\left\{\int_{x_{r}}^{+\infty} x_{r}^{p} x_{s}^{q-1} f\left(x_{r}\right)\left[F\left(x_{r}\right)\right]^{r-1} \times\right. \\
& \left.\left[F\left(x_{s}\right)-F\left(x_{r}\right)\right]^{s-r-1}\left[1-F\left(x_{s}\right)\right]^{n-s+1} d x_{s}\right\} d x_{r} .
\end{aligned}
$$

Since the PA distribution implies that $\theta^{2} \beta x^{2 \beta-1}[1-$ $F(x)]=\left(1+\theta x^{\beta}\right) f(x)$ for $x>0$, we get

$$
\begin{aligned}
& x_{r}^{p} x_{s}^{q-1} f\left(x_{r}\right)\left[F\left(x_{r}\right)\right]^{r-1}\left[F\left(x_{s}\right)-F\left(x_{r}\right)\right]^{s-r-1} \times \\
& {\left[1-F\left(x_{s}\right)\right]^{n-s+1}} \\
& =\frac{1}{\theta^{2} \beta} x_{r}^{p} x_{s}^{q-2 \beta} f\left(x_{r}\right)\left[F\left(x_{r}\right)\right]^{r-1} \times \\
& {\left[F\left(x_{s}\right)-F\left(x_{r}\right)\right]^{s-r-1}\left[1-F\left(x_{s}\right)\right]^{n-s} \times} \\
& \left\{\theta^{2} \beta x_{s}^{2 \beta-1}\left[1-F\left(x_{s}\right)\right]\right\} \\
& =\frac{1}{\theta^{2} \beta} x_{r}^{p} x_{s}^{q-2 \beta} f\left(x_{r}\right)\left[F\left(x_{r}\right)\right]^{r-1} \times \\
& {\left[F\left(x_{s}\right)-F\left(x_{r}\right)\right]^{s-r-1}\left[1-F\left(x_{s}\right)\right]^{n-s}\left(1+\theta x_{s}^{\beta}\right) f\left(x_{s}\right)} \\
& =\frac{1}{\theta^{2} \beta}\left\{x_{r}^{p} x_{s}^{q-2 \beta} f\left(x_{r}\right)\left[F\left(x_{r}\right)\right]^{r-1} \times\right. \\
& {\left[F\left(x_{s}\right)-F\left(x_{r}\right)\right]^{s-r-1}\left[1-F\left(x_{s}\right)\right]^{n-s} f\left(x_{s}\right)+} \\
& +\theta x_{r}^{p} x_{s}^{q-\beta} f\left(x_{r}\right)\left[F\left(x_{r}\right)\right]^{r-1}\left[F\left(x_{s}\right)-F\left(x_{r}\right)\right]^{s-r-1} \times \\
& \left.\left[1-F\left(x_{s}\right)\right]^{n-s}\left(1+\theta x_{s}^{\beta}\right) f\left(x_{s}\right)\right\} \\
& =\frac{1}{\theta^{2} \beta}\left\{x_{r}^{p} x_{s}^{q-2 \beta} C_{r, s: n}^{-1} f_{r, s: n}\left(x_{r}, x_{s}\right)\right. \\
& \left.+\theta x_{r}^{p} x_{s}^{q-\beta} C_{r, s: n}^{-1} f_{r, s: n}\left(x_{r}, x_{s}\right)\right\} .
\end{aligned}
$$

Therefore, by putting Equations (4) and (5) together, we obtain

$$
\begin{aligned}
& \mu_{r, s: n}^{p, q}-\mu_{r, s-1: n}^{p, q}=\frac{q}{n-s+1} \frac{1}{\theta^{2} \beta} \times \\
& {\left[\int_{-\infty}^{+\infty}\left\{\int_{x_{r}}^{+\infty} x_{r}^{p} x_{s}^{q-2 \beta} f_{r, s: n}\left(x_{r}, x_{s}\right) d x_{s}\right\} d x_{r}\right.} \\
& \left.+\theta \int_{-\infty}^{+\infty}\left\{\int_{x_{r}}^{+\infty} x_{r}^{p} x_{s}^{q-\beta} f_{r, s: n}\left(x_{r}, x_{s}\right) d x_{s}\right\} d x_{r}\right] \\
& =\frac{q}{n-s+1} \frac{1}{\theta^{2} \beta}\left[\mu_{r, s: n}^{p, q-2 \beta}+\theta \mu_{r, s: n}^{p, q-\beta}\right] .
\end{aligned}
$$

The stated result is here.

\subsection{Reversed order statistics}

The preceding theory can be applied to so-called reversed order statistics, which appear when $X_{1}, \ldots, X_{n}$ are arranged in decreasing order (see [14, Chapter 2.11]). That is, by denoting them sas $X_{1\left(r_{e}\right): n}, X_{2\left(r_{e}\right): n}, \ldots, X_{n\left(r_{e}\right): n}$, they satisfy $P\left(X_{1\left(r_{e}\right): n} \geq X_{2\left(r_{e}\right): n} \geq \ldots \geq X_{n\left(r_{e}\right): n}\right)=1$. They are useful for modeling phenomena involving data in descending order of magnitude, such as student grades from highest to lowest, city population in millions, and so on. For any $r=1,2, \ldots, n$, it is certain that $X_{r\left(r_{e}\right): n}=X_{n-r+1: n}$. Therefore, 
in full generality, the pdf of $X_{r\left(r_{e}\right): n}$ is given as $f_{r\left(r_{e}\right): n}(x)=f_{n-r+1: n}(x)$, that is

$$
\begin{aligned}
& f_{r\left(r_{e}\right): n}(x)=C_{r: n} f(x)[F(x)]^{n-r}[1-F(x)]^{r-1}, \\
& x \in \mathbb{R} .
\end{aligned}
$$

Hence, in the setting of the PA distribution, it comes

$$
\begin{aligned}
& f_{r\left(r_{e}\right): n}(x)=C_{r: n} \theta^{2} \beta x^{2 \beta-1} \times \\
& {\left[1-\left(1+\theta x^{\beta}\right) e^{-\theta x^{\beta}}\right]^{n-r}\left(1+\theta x^{\beta}\right)^{r-1} e^{-r \theta x^{\beta}}, \times} \\
& x>0
\end{aligned}
$$

and $f_{r\left(r_{e}\right): n}(x)=0$ for $x \leq 0$.

Moreover, the general expression of the joint pdf of $\left(X_{r\left(r_{e}\right): n}, X_{s\left(r_{e}\right): n}\right)$ for $r \leq s$ is given as $f_{r\left(r_{e}\right), s\left(r_{e}\right): n}\left(x_{r}, x_{s}\right)=f_{n-s+1, n-r+1: n}\left(x_{s}, x_{r}\right)$ for $x_{s}<x_{r}$, with $\left(x_{r}, x_{s}\right) \in \mathbb{R}^{2}$, that is

$$
\begin{aligned}
& f_{r\left(r_{e}\right), s\left(r_{e}\right): n}\left(x_{r}, x_{s}\right)=C_{r, s: n} f\left(x_{r}\right) f\left(x_{s}\right) \times \\
& {\left[F\left(x_{s}\right)\right]^{n-s}\left[F\left(x_{r}\right)-F\left(x_{s}\right)\right]^{s-r-1}\left[1-F\left(x_{r}\right)\right]^{r-1},} \\
& x_{s}<x_{r},
\end{aligned}
$$

with $\left(x_{r}, x_{s}\right) \in \mathbb{R}^{2}$. In the context of the PA distribution, it comes

$$
\begin{aligned}
& f_{r\left(r_{e}\right), s\left(r_{e}\right): n}\left(x_{r}, x_{s}\right)=C_{r, s: n} \theta^{4} \beta^{2} x_{r}^{2 \beta-1} x_{s}^{2 \beta-1} \times \\
& e^{-\theta x_{s}^{\beta}}\left[1-\left(1+\theta x_{s}^{\beta}\right) e^{-\theta x_{s}^{\beta}}\right]^{n-s} \times \\
& {\left[\left(1+\theta x_{s}^{\beta}\right) e^{-\theta x_{s}^{\beta}}-\left(1+\theta x_{r}^{\beta}\right) e^{-\theta x_{r}^{\beta}}\right]^{s-r-1} \times} \\
& \left(1+\theta x_{r}^{\beta}\right)^{r-1} e^{-r \theta x_{r}^{\beta}}, \quad x_{r}>x_{s}>0,
\end{aligned}
$$

and $f_{r\left(r_{e}\right), s\left(r_{e}\right): n}\left(x_{r}, x_{s}\right)=0$ when $x_{r}>x_{s}>0$ is not satisfied.

The following proposition presents moments results on reversed order statistics for the PA distribution.

Proposition 2.4 Let $p$ and $q$ be positive integers, $\mu_{r\left(r_{e}\right): n}^{p}=E\left(X_{r\left(r_{e}\right): n}^{p}\right)$ be the pth moment of $X_{r\left(r_{e}\right): n}$ and $\mu_{r\left(r_{e}\right), s\left(r_{e}\right): n}^{p, q}=E\left(X_{r\left(r_{e}\right): n}^{p} X_{s\left(r_{e}\right): n}^{q}\right)$ be the $(p, q)$ th product moment of $\left(X_{r\left(r_{e}\right): n}, X_{s\left(r_{e}\right): n}\right)$.

- The exact expression of $\mu_{r\left(r_{e}\right): n}^{p}$ is

$$
\begin{aligned}
& \mu_{r\left(r_{e}\right): n}^{p}=C_{r: n} \theta^{-p / \beta} \sum_{k=0}^{n-r} \sum_{v=0}^{k+r-1}\left(\begin{array}{c}
n-r \\
k
\end{array}\right) \times \\
& \left(\begin{array}{c}
k+r-1 \\
v
\end{array}\right)(-1)^{k} \frac{1}{(k+r)^{p / \beta+2+v}} \times \\
& \Gamma\left(\frac{p}{\beta}+2+v\right) .
\end{aligned}
$$

- The following recurrence relationship holds:

$$
\begin{aligned}
& \mu_{r\left(r_{e}\right): n}^{p}= \\
& \mu_{r\left(r_{e}\right)-1: n}^{p}+\frac{p}{r} \frac{1}{\theta^{2} \beta}\left[\mu_{r\left(r_{e}\right): n}^{p-2 \beta}+\theta \mu_{r\left(r_{e}\right): n}^{p-\beta}\right] .
\end{aligned}
$$

- The following recurrence relationship holds:

$$
\begin{aligned}
& \mu_{r\left(r_{e}\right), s\left(r_{e}\right): n}^{p, q}=\mu_{r\left(r_{e}\right), s\left(r_{e}\right)-1: n}^{p, q}+ \\
& \frac{p}{r} \frac{1}{\theta^{2} \beta}\left[\mu_{r\left(r_{e}\right), s\left(r_{e}\right): n}^{p, q-2 \beta}+\theta \mu_{r\left(r_{e}\right), s\left(r_{e}\right): n}^{p, q-\beta}\right] .
\end{aligned}
$$

Proof: The proof of the first item is derived from the following formula:

$$
\mu_{r\left(r_{e}\right): n}^{p}=E\left(X_{n-r+1: n}^{p}\right)=\mu_{n-r+1: n}^{p}
$$

and Proposition 2.1, this proposition is thus applied with $n-r+1$ instead of $r$. The two other items are deduced from the following formula:

$$
\begin{aligned}
& \mu_{r\left(r_{e}\right), s\left(r_{e}\right): n}^{p, q}=E\left(X_{n-r+1: n}^{p} X_{n-s+1: n}^{q}\right) \\
& =E\left(X_{n-s+1: n}^{q} X_{n-r+1: n}^{p}\right)=\mu_{n-s+1, n-r+1: n}^{q, p},
\end{aligned}
$$

where the inequality $X_{n-s+1: n} \leq X_{n-r+1: n}$ has been taken into account, and Propositions 2.2 and 2.3 , respectively. The stated result is established.

To our knowledge, the general formulas on moments of reverse order statistics used in the proof of Proposition 2.4 are novel in the literature and can be reused for other purposes.

\section{Upper record statistics}

All the general formulas used in this section can be found in [14, Chapter 3].

\subsection{Univariate case}

Records play an important role in real-world applications such as data from weather stations, sporting data, economic data, and strength data. Chandler's seminal work (see [10]) raises the possibility of using upper record statistics in statistical inference. The upper record statistics' mathematical context is described below. Let us consider $\left(X_{n}\right)_{n \geq 1}$ a sequence of independent random variables with the same distribution. Let us denote by $F(x)$ and $f(x)$ the related cdf and pdf of this common distribution, respectively, and $X_{i: n}$ be the $i$ th order statistic as described in the previous section. For fixed $k \geq 1$, we introduce $U_{k}(n)$ for $n \geq 1$ by

$$
\begin{aligned}
& U_{k}(n+1)= \\
& \min \left\{j>U_{k}(n): X_{j: j+k-1}>X_{U_{k}(n): U_{k}(n)+k-1}\right\}
\end{aligned}
$$


for $n \geq 2$, and $U_{k}(1)=1$. Then, we set

$$
Y_{n}^{(k)}=X_{U_{k}(n): U_{k}(n)+k-1}, \quad n \geq 1 .
$$

The random variable $Y_{n}^{(k)}$ is called the $k$ th upper record statistic. Then, in full generality, the pdf of $Y_{n}^{(k)}$ is readily written as

$$
f_{Y_{n}^{(k)}}(x)=\frac{k^{n}}{(n-1) !}[R(x)]^{n-1}[1-F(x)]^{k-1} f(x),
$$$$
x \in \mathbb{R},
$$

where $R(x)=-\ln [1-F(x)]$, corresponding to the cumulative hazard rate function related to $F(x)$.

In the context of the PA distribution, it is given as

$$
\begin{aligned}
& f_{Y_{n}^{(k)}}(x)=\frac{\theta^{2} \beta k^{n}}{(n-1) !} x^{2 \beta-1}\left(1+\theta x^{\beta}\right)^{k-1} e^{-k \theta x^{\beta}} \times \\
& {\left[\theta x^{\beta}-\ln \left(1+\theta x^{\beta}\right)\right]^{n-1}, \quad x>0,}
\end{aligned}
$$

and $f_{Y_{n}^{(k)}}(x)=0$ for $x \leq 0$.

To the best of our knowledge, there is no equivalent in the literature, and it can be easily expanded by assuming that $n$ and $k$ are real numbers greater than 1.

It is difficult to have an explicit expression for the moment of $Y_{n}^{(k)}$ due to the logarithmic term. As a result, we use the knowledge from lower order moments to compute moments of special order for upper record statistics in the spirit of defining recurrence connections.

The following proposition shows that the moments of $Y_{n}^{(k)}$ are linked by a simple recurrence relationship.

Proposition 3.1 Let $p$ be a positive integer and $\mu_{K(n)}^{p}=E\left(\left[Y_{n}^{(k)}\right]^{p}\right)$ be the pth moment of $Y_{n}^{(k)}$. Then, the following recurrence relationship holds:

$$
\mu_{K(n)}^{p}=\mu_{K(n-1)}^{p}+\frac{1}{\theta^{2} \beta} \frac{p}{k}\left[\mu_{K(n)}^{p-2 \beta}+\theta \mu_{K(n)}^{p-\beta}\right] .
$$

Proof: We begin by invoking [14, Theorem 3.1, Equation (3.24)], result initially established by [15]. It can be formulated as

$$
\begin{aligned}
& \mu_{K(n)}^{p}-\mu_{K(n-1)}^{p}= \\
& \frac{p k^{n-1}}{(n-1) !} \int_{-\infty}^{+\infty} x^{p-1}[1-F(x)]^{k}[R(x)]^{n-1} d x .
\end{aligned}
$$

By using the following relationship inherent to the PA distribution: $\theta^{2} \beta x^{2 \beta-1}[1-F(x)]=\left(1+\theta x^{\beta}\right) f(x)$ for $x>0$, it comes

$$
\begin{aligned}
& x^{p-1}[1-F(x)]^{k}=\frac{1}{\theta^{2} \beta} x^{p-2 \beta}[1-F(x)]^{k-1} \times \\
& \left\{\theta^{2} \beta x^{2 \beta-1}[1-F(x)]\right\} \\
& =\frac{1}{\theta^{2} \beta} x^{p-2 \beta}[1-F(x)]^{k-1}\left(1+\theta x^{\beta}\right) f(x) \\
& =\frac{1}{\theta^{2} \beta}\left[x^{p-2 \beta}[1-F(x)]^{k-1} f(x)+\right. \\
& \left.\theta x^{p-\beta}[1-F(x)]^{k-1} f(x)\right] .
\end{aligned}
$$

Hence

$$
\begin{aligned}
& \frac{p k^{n-1}}{(n-1) !} x^{p-1}[1-F(x)]^{k}[R(x)]^{n-1}= \\
& \frac{1}{\theta^{2} \beta} \frac{p}{k}\left[x^{p-2 \beta} \frac{k^{n}}{(n-1) !}[R(x)]^{n-1}[1-F(x)]^{k-1} f(x)\right. \\
& \left.+\theta x^{p-\beta} \frac{k^{n}}{(n-1) !}[R(x)]^{n-1}[1-F(x)]^{k-1} f(x)\right] \\
& =\frac{1}{\theta^{2} \beta} \frac{p}{k}\left[x^{p-2 \beta} f_{Y_{n}^{(k)}}(x)+\theta x^{p-\beta} f_{Y_{n}^{(k)}}(x)\right]
\end{aligned}
$$

It follows from Equations (6) and (8) that

$$
\begin{aligned}
& \mu_{K(n)}^{p}-\mu_{K(n-1)}^{p}= \\
& \frac{1}{\theta^{2} \beta} \frac{p}{k}\left[\int_{0}^{+\infty} x^{p-2 \beta} f_{Y_{n}^{(k)}}(x) d x\right. \\
& \left.+\theta \int_{0}^{+\infty} x^{p-\beta} f_{Y_{n}^{(k)}}(x) d x\right] \\
& =\frac{1}{\theta^{2} \beta} \frac{p}{k}\left[\mu_{K(n)}^{p-2 \beta}+\theta \mu_{K(n)}^{p-\beta}\right] .
\end{aligned}
$$

The desired result is established.

\subsection{Bivariate case}

We now explore the bivariate case. First, the general expression of the joint pdf of $\left(Y_{m}^{(k)}, Y_{n}^{(k)}\right)$ with $n \leq m$ is specified as

$$
\begin{aligned}
& f_{\left(Y_{m}^{(k)}, Y_{n}^{(k)}\right)}\left(x_{m}, x_{n}\right)= \\
& \frac{k^{n}}{(m-1) !(n-m-1) !} r\left(x_{m}\right) f\left(x_{n}\right)\left[R\left(x_{m}\right)\right]^{m-1} \times \\
& {\left[R\left(x_{n}\right)-R\left(x_{m}\right)\right]^{n-m-1}\left[1-F\left(x_{n}\right)\right]^{k-1},} \\
& x_{m}<x_{n},
\end{aligned}
$$

with $\left(x_{m}, x_{n}\right) \in \mathbb{R}^{2}$, where $r(x)=f(x) /[1-F(x)]$ denotes the hazard rate function related to $F(x)$. In 
the setting of the PA distribution, we get

$$
\begin{aligned}
& f_{\left(Y_{m}^{(k)}, Y_{n}^{(k)}\right)}\left(x_{m}, x_{n}\right)=\frac{k^{n}}{(m-1) !(n-m-1) !} \times \\
& \theta^{4} \beta^{2} \frac{x_{m}^{2 \beta-1}}{1+\theta x_{m}^{\beta}} x_{n}^{2 \beta-1}\left[\theta x_{m}^{\beta}-\ln \left(1+\theta x_{m}^{\beta}\right)\right]^{m-1} \times \\
& {\left[\theta\left(x_{n}^{\beta}-x_{m}^{\beta}\right)+\ln \left(\frac{1+\theta x_{m}^{\beta}}{1+\theta x_{n}^{\beta}}\right)\right]^{n-m-1} \times} \\
& \left(1+\theta x_{n}^{\beta}\right)^{k-1} e^{-k \theta x_{n}^{\beta}}, \quad x_{n}>x_{m}>0,
\end{aligned}
$$

and $f_{\left(Y_{m}^{(k)}, Y_{n}^{(k)}\right)}\left(x_{m}, x_{n}\right)=0$ when $x_{n}>x_{m}>0$ is not satisfied.

The following result establishes a relationship between the product moments of the $k$ th upper record statistic.

Proposition 3.2 Let $p$ and $q$ be positive integers and $\mu_{K(m, n)}^{p, q}=E\left(\left[Y_{m}^{(k)}\right]^{p}\left[Y_{n}^{(k)}\right]^{q}\right)$ be the $(p, q)$ th product moment of $\left(Y_{m}^{(k)}, Y_{n}^{(k)}\right)$. Then, $\mu_{r, s: n}^{p, q}$ satisfies the following relationship:

$\mu_{K(m, n)}^{p, q}=\mu_{K(m, n-1)}^{p, q}+\frac{1}{\theta^{2} \beta} \frac{q}{k}\left[\mu_{K(m, n)}^{p, q-2 \beta}+\theta \mu_{K(m, n)}^{p, q-\beta}\right]$.

Proof: By virtue of [14, Theorem 3.1, Equation (3.25)], we have

$$
\begin{aligned}
& \mu_{K(m, n)}^{p, q}-\mu_{K(m, n-1)}^{p, q}=\frac{q k^{n-1}}{(m-1) !(n-m-1) !} \times \\
& \int_{-\infty}^{+\infty}\left\{\int_{x_{m}}^{+\infty} x_{m}^{p} x_{n}^{q-1} r\left(x_{m}\right)\left[R\left(x_{m}\right)\right]^{m-1} \times\right. \\
& \left.\left[R\left(x_{n}\right)-R\left(x_{m}\right)\right]^{n-m-1}\left[1-F\left(x_{n}\right)\right]^{k} d x_{n}\right\} d x_{m} .
\end{aligned}
$$

For the PA distribution precisely, proceeding as in Equation (7), we get

$$
\begin{aligned}
& x_{n}^{q-1}\left[1-F\left(x_{n}\right)\right]^{k}= \\
& \frac{1}{\theta^{2} \beta}\left[x_{n}^{q-2 \beta}\left[1-F\left(x_{n}\right)\right]^{k-1} f\left(x_{n}\right)\right. \\
& \left.+\theta x_{n}^{q-\beta}\left[1-F\left(x_{n}\right)\right]^{k-1} f\left(x_{n}\right)\right] .
\end{aligned}
$$

Therefore

$$
\begin{aligned}
& \frac{q k^{n-1}}{(m-1) !(n-m-1) !} x_{m}^{p} x_{n}^{q-1} r\left(x_{m}\right) \times \\
& {\left[R\left(x_{m}\right)\right]^{m-1}\left[R\left(x_{n}\right)-R\left(x_{m}\right)\right]^{n-m-1}\left[1-F\left(x_{n}\right)\right]^{k}} \\
& =\frac{1}{\theta^{2} \beta} \frac{q}{k}\left[x_{m}^{p} x_{n}^{q-2 \beta} \frac{k^{n}}{(m-1) !(n-m-1) !} r\left(x_{m}\right) \times\right. \\
& {\left[R\left(x_{m}\right)\right]^{m-1}\left[R\left(x_{n}\right)-R\left(x_{m}\right)\right]^{n-m-1} \times} \\
& {\left[1-F\left(x_{n}\right)\right]^{k-1} f\left(x_{n}\right)+} \\
& \theta x_{m}^{p} x_{n}^{q-\beta} \frac{k^{n}}{(m-1) !(n-m-1) !} r\left(x_{m}\right)\left[R\left(x_{m}\right)\right]^{m-1} \times \\
& \left.\left[R\left(x_{n}\right)-R\left(x_{m}\right)\right]^{n-m-1}\left[1-F\left(x_{n}\right)\right]^{k-1} f\left(x_{n}\right)\right] \\
& =\frac{1}{\theta^{2} \beta} \frac{q}{k}\left[x_{m}^{p} x_{n}^{q-2 \beta} f_{\left(Y_{m}^{(k)}, Y_{n}^{(k)}\right)}\left(x_{m}, x_{n}\right)\right. \\
& \left.+\theta x_{m}^{p} x_{n}^{q-\beta} f_{\left(Y_{m}^{(k)}, Y_{n}^{(k)}\right)}\left(x_{m}, x_{n}\right)\right] .
\end{aligned}
$$

The combination of Equations (10) and (9) gives

$$
\begin{aligned}
& \mu_{K(m, n)}^{p, q}-\mu_{K(m, n-1)}^{p, q}=\frac{1}{\theta^{2} \beta} \frac{q}{k} \times \\
& {\left[\int _ { - \infty } ^ { + \infty } \left\{\int_{x_{m}}^{+\infty} x_{m}^{p} x_{n}^{q-2 \beta} \times\right.\right.} \\
& \left.f_{\left(Y_{m}^{(k)}, Y_{n}^{(k)}\right)}\left(x_{m}, x_{n}\right) d x_{n}\right\} d x_{m} \\
& +\theta \int_{-\infty}^{+\infty}\left\{\int_{x_{m}}^{+\infty} x_{m}^{p} x_{n}^{q-\beta} \times\right. \\
& \left.\left.f_{\left(Y_{m}^{(k)}, Y_{n}^{(k)}\right)}\left(x_{m}, x_{n}\right) d x_{n}\right\} d x_{m}\right] \\
& =\frac{1}{\theta^{2} \beta} \frac{q}{k}\left[\mu_{K(m, n)}^{p, q-2 \beta}+\theta \mu_{K(m, n)}^{p, q-\beta}\right] .
\end{aligned}
$$

The desired result is established.

The findings for single and product moment of order statistics, as well as upper record statistics for the PA distribution, may be used for a variety of statistical reasons, which we can leave to future research.

\section{Acknowledgments}

The authors would like to warmly thank the reviewer for constructive comments on the paper.

\section{References:}

[1] Lv, H.Q., Gao, L.H. and Chen, C.L. (2002). Ailamujia distribution and its application in supportability data analysis, Journal of Academy of Armored Force Engineering, 16, 48-52. 
[2] Ul Ain, S.Q., Aijaz, A. and Tripathi, R. (2020). A new two parameter Ailamujia distribution with applications in bio-medicine, Journal of Xi'an University of Architecture \& Technology, 12, 11, 592-604.

[3] Jamal, F., Chesneau, C., Aidi, K. and Ali, A. (2021). Theory and application of the power Ailamujia distribution, Journal of Mathematical Modeling, 9, 3, 391-413.

[4] Khan, A.H., Yaqub, M. and Parvez, S. (1983a). Recurrence relations between moments of order statistics, Naval Research Logistics Quarterly, 30, 419-441.

[5] Khan, A.H., Parvez, S. and Yaqub, M. (1983b). Recurrence relations between product moments of order statistics, Journal of Statistical Planning and Inference, 8, 175-183.

[6] Raqab, M.Z. (2004). Generalized exponential distribution: moments of order statistics, Statistics $38,1,29-41$.

[7] Thomas, P.Y. and Samuel, P. (2008). Recurrence relations for the moments of order statistics from a beta distribution, Statistical Papers, 49, 139146.

[8] Saran, J., Verma, K. and Pushkarna, N. (2018). Relationships for moments of generalized order statistics from Erlang-truncated exponential distribution and related inference, ProbStat Forum, 11, 91-103.

[9] Sultan, K.S. and AL-Thubyani, W.S. (2016). Higher order moments of order statistics from the Lindley distribution and associated inference, Journal of Statistical Computation and Simulation, 86, 17, 3432-3445.
[10] Chandler, K.N. (1952). The distribution and frequency of record values, Journal of the Royal Statistical Society: Series B, 14, 220-228.

[11] Saran, J. and Pushkarna, N. (2000). Relationships for moments of record values from linearexponential distribution, Journal of Applied Statistical Science, 10, 69-76.

[12] Saran, J. and Singh, S.K. (2008). Recurrence relations for single and product moments of $\mathrm{k}$-th record values from linear-exponential distribution and a characterization, Asian Journal of Mathematics and Statistics, 1, 3, 159-164.

[13] Sultan, K.S. (2007). Record values from the modified Weibull distribution, International Mathematical Forum, 2, 41, 2045-2054.

[14] Shahbaz, M.Q., Ahsanullah, M., Hanif Shahbaz, S and Al-Zahrani, B. (2016). Ordered Random Variables: Theory and Applications, Atlantis Press and Springer, France.

[15] Bieniek, M. and Szynal, D. (2002). Recurrence relations for distribution functions and moments of kth record values, Journal of Mathematical Sciences, 111, 3, 3511-3519.

\section{Creative Commons Attribution License 4.0 (Attribution 4.0 International , CC BY 4.0)}

This article is published under the terms of the Creative Commons Attribution License 4.0

https://creativecommons.org/licenses/by/4.0/deed.en US 